\title{
SURFACES WITH METRIC AND CURVATURE TENSORS THAT DEPEND ON ONE COORDINATE ONLY ARE GENERAL HELICOIDS*
}

\author{
BY JAMES G. SIMMONDS (University of Virginia, Charlottesville)
}

1. Introduction. The assertion in the title was motivated by and answers the following question in nonlinear first-approximation shell theory: what midsurfaces admit extensional and bending strains that are functions of one variable only? As these strains depend on the differences $\bar{a}-\mathbf{a}$ and $\bar{b}-\mathrm{b}$ of the metric and curvature tensors of the deformed and undeformed midsurfaces, our result implies that both midsurfaces must be (modulo a rigid body displacement) general helicoids, i.e. have a vector parametric representation of the form

$$
\mathbf{r}(\phi, \theta)=r(\phi)(\mathrm{i} \cos \theta+\mathrm{j} \sin \theta)+[\zeta(\phi)+a \theta] \mathbf{k},
$$

where $(\mathbf{i}, \mathbf{j}, \mathbf{k})$ are the conventional orthonormal Cartesian base vectors, $r$ and $\zeta$ are arbitrary functions of the parameter $\phi$, and $a$ is a constant. If the thickness and elastic parameters of the shell depend, at most, on $\phi$ only, then the field equations reduce to ordinary differential equations. Numerous writers have considered shells of revolution, homogeneous in the circumferential direction, undergoing purely axisymmetric or purely torsional nonlinear displacements. Such situations obviously lead to ordinary differential equations. Apparently, the only work on other nonlinear shell problems leading to onedimensional strains (other than several papers on inextensional deformations) is Reissner's study of shallow, right helicoids [1], and an unpublished manuscript by Wan on nonshallow right helicoids [2].

Eisenhart states that any surface with a metric tensor depending on one surface coordinate only is applicable to a surface of revolution [3, p. 149] and quotes a theorem of Bour that any helicoid is applicable to a surface of revolution [3, p. 147]. Furthermore, Eisenhart establishes some theorems, based on Weingarten's work, for general surfaces applicable to surfaces of revolution [3, p. 362-364]. However, he does not consider surfaces with a curvature tensor depending on the same single variable as does the metric tensor of the applicable surface of revolution. Rather than attempt to specialize Eisenhart's analysis to obtain our result, we shall proceed in a simpler, more direct way.

2. Analysis. Let $\mathbf{r}\left(\xi^{\alpha}\right), \alpha=1,2$, denote the equation of a smooth surface $S$. The covariant base vectors are $\mathbf{a}_{\alpha}=\mathbf{r}_{, \alpha} \equiv \partial \mathbf{r} / \partial \xi^{\alpha}$ and the covariant components of the metric tensor a are $a_{\alpha \beta}=\mathbf{a}_{\alpha} \cdot \mathbf{a}_{\beta}$. The unit normal on $S$ is $\mathbf{n}=\frac{1}{2} \epsilon^{\alpha \beta} \mathbf{a}_{\alpha} \times \mathbf{a}_{\beta}$, where $\epsilon^{\alpha \beta \beta}$ are the contravariant components of the permutation tensor. The covariant components of the curvature tensor $\mathbf{b}$ are $b_{\alpha \beta}=\mathbf{n} \cdot \mathbf{r}, \alpha \beta$.

Let $\xi^{\alpha}(s)$ denote the parametric equations of an arbitrary curve $C$ on $S$, where $s$ is arc length. Then

$$
\mathrm{t}=\mathbf{r}^{\prime}\left(\xi^{\alpha}(s)\right)=\mathbf{r}_{\alpha} d \xi^{\alpha} / d s \equiv \mathrm{a}_{\alpha} t^{\alpha}
$$

\footnotetext{
* Received April 24, 1978. This research was supported by the National Science Foundation under grant MPS-73-08659A02.
} 
is a unit tangent vector along $C$. If

$$
\mathbf{u}=\mathbf{n} \times \mathfrak{t}=\epsilon_{\alpha \beta} \mathbf{a}^{\beta} t^{\alpha},
$$

then we have well-known Frenet formulas for a curve on a surface $[4$, p. 202];

$$
\begin{aligned}
\mathbf{t}^{\prime} & =\kappa_{\mathbf{g}} \mathbf{u}+\kappa_{n} \mathrm{n}, \\
\mathbf{u}^{\prime} & =-\kappa_{\mathbf{g}} \mathrm{t}-\tau_{\mathbf{g}} \mathrm{n}, \\
\mathbf{n}^{\prime} & =-\kappa_{n} \mathrm{t}+\tau_{\mathbf{g}} \mathbf{u},
\end{aligned}
$$

where $\kappa_{\mathrm{g}}, \kappa_{n}$, and $\tau_{\mathrm{g}}$ are, respectively, the geodesic curvature, the normal curvature, and the geodesic torsion of $C$. F rom (2) to (6), and the Gauss-Weingarten equations

$$
\mathbf{a}_{\alpha, \beta}=\Gamma_{\alpha \beta}^{\gamma} \mathbf{a}_{\gamma}+b_{\alpha \beta} \mathbf{n}, \quad \mathbf{n}_{, \alpha}=-b_{\alpha}^{\beta} \mathbf{a}_{\beta},
$$

follows

$$
\kappa_{n}=b_{\alpha \beta} t^{\alpha} t^{\beta}, \quad \kappa_{\mathrm{g}}=\left.\epsilon_{\alpha \beta} t^{\beta}\right|_{\gamma} t^{\alpha} t^{\gamma}, \quad \tau_{\mathrm{g}}=\epsilon_{\alpha \beta} b_{\gamma}^{\alpha} t^{\beta} t^{\gamma},
$$

where $\Gamma_{\alpha \beta}^{\gamma}$ are the Christoffel symbols and

$$
\left.t^{\beta}\right|_{\gamma}=t_{, \gamma}^{\beta}+\Gamma_{\gamma \lambda}^{\beta} t^{\lambda} .
$$

Now let $\xi=\xi^{1}, \eta=\xi^{2}$, let $C$ coincide with the $\xi$-coordinate curve, and assume that a and $\mathrm{b}$ depend on $\eta$ only. Then $t^{1}=a_{11}^{-1 / 2}(\eta), t^{2}=0$ and $\kappa_{n}, \kappa_{\mathrm{g}}$, and $\tau_{\mathrm{g}}$ reduce to functions of $\eta$ only. Furthermore, $f^{\prime}=f_{, \xi} a_{11}^{-1 / 2}(\eta)$, so that $\eta$ enters the Frenet equations (4) to (6) only as a parameter. That is, (4) to (6) are, formally constant coefficient ordinary differential equations in $\xi$. This implies that the general solution for $t$ is of the form

$$
\mathfrak{t}(\xi, \eta)=\mathbf{f}(\eta) \cos \lambda(\eta) \xi+\mathbf{g}(\eta) \sin \lambda(\eta) \xi+\mathbf{h}(\eta) .
$$

Since $\mathbf{r}_{\xi}=a_{11}^{1 / 2}(\eta) \mathbf{t}$, it follows that $\mathbf{r}$ has the general form

$$
\mathbf{r}(\xi, \eta)=\mathbf{x}(\eta) \cos \lambda(\eta) \xi+\mathbf{y}(\eta) \sin \lambda(\eta) \xi+\mathbf{z}(\eta)+\xi \mathbf{w}(\eta),
$$

where, at this stage, $\mathbf{w}, \mathbf{x}, \mathbf{y}, \mathbf{z}$, and $\lambda$ are arbitrary. To further restrict these quantities, we require that $a_{\alpha \beta}$ depend on $\eta$ only. It turns out that this also automatically makes $b_{\alpha \beta}$ a function of $\eta$ only.

From (11),

$$
\mathbf{r}_{, \xi} \equiv \mathbf{r}^{\bullet}=-\lambda \mathbf{x} \sin \lambda \xi+\lambda \mathbf{y} \cos \lambda \xi+\mathbf{w}
$$

Hence

$$
\begin{aligned}
a_{11}= & \mathbf{r}^{\bullet} \cdot \mathbf{r}^{\bullet}=\frac{1}{2} \lambda^{2}(\mathbf{x} \cdot \mathbf{x}+\mathbf{y} \cdot \mathbf{y})+\mathbf{w} \cdot \mathbf{w} \\
& +2 \lambda \mathbf{y} \cdot \mathbf{w} \cos \lambda \xi-2 \lambda \mathbf{x} \cdot \mathbf{w} \sin \lambda \xi+\frac{1}{2} \lambda^{2}(\mathbf{y} \cdot \mathbf{y}-\mathbf{x} \cdot \mathbf{x}) \cos 2 \lambda \xi-\lambda^{2} \mathbf{x} \cdot \mathbf{y} \sin 2 \lambda \xi .
\end{aligned}
$$

Excluding the trivial case $\lambda=0$, we see that the right side of (13) will be independent of $\xi$ only if

$$
w \cdot x=w \cdot y=x \cdot y=0, \quad x \cdot x=y \cdot y,
$$

i.e., only if $\mathbf{w}, \mathbf{x}$, and $\mathbf{y}$ are mutually orthogonal and $\mathbf{x}$ and $\mathbf{y}$ of the same length.

It is now convenient to set

$$
\mathbf{x}(\eta)=n(\eta) \mathbf{e}_{x}(\eta), \quad \mathbf{y}(\mathbf{n})=n(\eta) \mathbf{e}_{y}(\eta),
$$


where $\mathbf{e}_{x}$ and $\mathbf{e}_{y}$ are orthogonal unit vectors. Thereby, (11) may be cast into the form

$$
\mathbf{r}(\xi, \eta)=r(\eta) \mathbf{e}_{r}(\xi, \eta)+\mathbf{z}(\eta)+\xi \mathbf{w}(\eta)
$$

where

$$
\mathbf{e}_{r}(\xi, \eta)=\mathbf{e}_{x}(\eta) \cos \lambda(\eta) \xi+\mathbf{e}_{y} \sin \lambda(\eta) \xi
$$

Note that

$$
\begin{gathered}
\mathbf{e}_{r}^{\bullet}=\lambda\left(-\mathbf{e}_{x} \sin \lambda \xi+\mathbf{e}_{y} \cos \lambda \xi\right) \equiv \lambda \mathbf{e}_{\theta}, \\
\mathbf{e}_{r, \eta} \equiv \mathbf{e}_{r}^{\prime}=\xi \lambda^{\prime} \mathbf{e}_{\theta}+\mathbf{e}_{x}^{\prime} \cos \lambda \xi+\mathbf{e}_{y}^{\prime} \sin \lambda \xi,
\end{gathered}
$$

so from (16), (18), and (19),

$$
\begin{gathered}
\mathbf{r}^{*}=\lambda r \mathbf{e}_{\theta}+\mathbf{w}, \\
\mathbf{r}^{\prime}=r^{\prime} \mathbf{e}_{r}+r\left(\xi \lambda^{\prime} \mathbf{e}_{\theta}+\mathbf{e}_{x}^{\prime} \cos \lambda \xi+\mathbf{e}_{y}^{\prime} \sin \lambda \xi\right)+\mathbf{z}^{\prime}+\xi \mathbf{w}^{\prime} .
\end{gathered}
$$

Taking the dot product of (20) with (21), breaking out the $\xi$-dependence of $e_{r}$ and $e_{\theta}$, and noting that $\mathbf{e}_{x} \cdot \mathbf{w}=\mathbf{e}_{y} \cdot \mathbf{w}=\mathbf{e}_{x}^{\prime} \cdot \mathbf{e}_{x}=\mathbf{e}_{y}^{\prime} \cdot \mathbf{e}_{y}=\mathbf{e}_{x}^{\prime} \cdot \mathbf{e}_{y}+\mathbf{e}_{x} \cdot \mathbf{e}_{y}^{\prime}=0$, we find that

$$
\begin{aligned}
a_{12}=\mathbf{r}^{\bullet} \cdot \mathbf{r}^{\prime} & =\mathbf{w} \cdot \mathbf{z}^{\prime}+\frac{1}{2} \lambda r^{2}\left(\mathbf{e}_{y} \cdot \mathbf{e}_{x}^{\prime}-\mathbf{e}_{x} \cdot \mathbf{e}_{y}^{\prime}\right)+\left(\mathbf{w} \cdot \mathbf{w}^{\prime}+r^{2} \lambda \lambda^{\prime}\right) \xi+r\left(\mathbf{w} \cdot \mathbf{e}_{x}^{\prime}+\lambda \mathbf{e}_{y} \cdot \mathbf{z}^{\prime}\right) \cos \lambda \xi \\
& +r\left(\mathbf{w} \cdot \mathbf{e}^{\prime}{ }_{y}-\lambda \mathbf{e}_{x} \cdot \mathbf{z}^{\prime}\right) \sin \lambda \xi \quad+\lambda r \mathbf{w}^{\prime} \cdot \mathbf{e}_{y} \xi \cos \lambda \xi-\lambda r \mathbf{w}^{\prime} \cdot \mathbf{e}_{x} \xi \sin \lambda \xi .
\end{aligned}
$$

The right side of (22) will be independent of $\xi$ only if

$$
\mathbf{w}^{\prime} \cdot \mathbf{e}_{x}=\mathbf{w}^{\prime} \cdot \mathbf{e}_{y}=\mathbf{z}^{\prime} \cdot \mathbf{e}_{x}=\mathbf{z}^{\prime} \cdot \mathbf{e}_{y}=\mathbf{w} \cdot \mathbf{W}^{\prime}+r^{2} \lambda \lambda^{\prime}=0 .
$$

We now distinguish two cases.

$\mathbf{w} \neq 0$. As $\mathbf{e}_{x} \cdot \mathbf{w}=\mathbf{e}_{y} \cdot \mathbf{w}=0$ from (14), the first two relations in (23) imply that

$$
\mathbf{w}^{\prime}=f(\eta) \mathbf{w}
$$

where $f(\eta)$ is arbitrary. This equation has a solution of the form

$$
\mathbf{w}=C \exp \int f(\eta) d \eta \mathbf{k} \equiv w(\eta) \mathbf{k},
$$

where $C$ is an arbitrary non-zero constant and $\mathbf{k}$ is a fixed unit vector.

Since $\mathbf{w}$ is proportional to $\mathbf{k}, \mathbf{e}_{x}$ and $\mathbf{e}_{y}$ must remain in a fixed plane and we may set

$$
\mathbf{e}_{x}=\mathbf{i} \cos \psi(\eta)+\mathbf{j} \sin \psi(\eta), \quad \mathbf{e}_{y}=-\mathbf{i} \sin \psi(\eta)+\mathbf{j} \cos \psi(\eta),
$$

where $\psi(\eta)$ is arbitrary. From (17) to (19) follows

$$
\mathbf{C}_{r}(\xi, \eta)=\mathbf{i} \cos [\lambda(\eta) \xi+\psi(\eta)]+\mathrm{j} \sin [\lambda(\eta) \xi+\psi(\eta)],
$$

and

$$
\mathbf{e}_{r}^{\bullet}=\lambda \mathbf{e}_{\theta}, \quad \mathbf{e}_{r}^{\prime}=\left(\lambda^{\prime} \xi+\psi^{\prime}\right) \mathbf{e}_{\theta} .
$$

The third and fourth relations in (23) imply that

$$
\mathbf{z}=z(\eta) \mathbf{k},
$$

where $z(\eta)$ is arbitrary. The fifth relation in (23) remains to be satisfied.

We now have

$$
\begin{aligned}
& \mathbf{r}(\xi, \eta)=r(\eta) \mathbf{e}_{r}(\xi, \eta)+[z(\eta)+\xi w(\eta)] \mathbf{k} \\
& \mathbf{r}^{\prime}=r^{\prime} \mathbf{e}_{r}+r\left(\lambda^{\prime} \xi+\psi^{\prime}\right) \mathbf{e}_{\theta}+\left(z^{\prime}+\xi w^{\prime}\right) \mathbf{k} .
\end{aligned}
$$


Hence,

$$
a_{22}=\mathbf{r}^{\prime} \cdot \mathbf{r}^{\prime}=r^{\prime 2}+r^{2} \psi^{\prime 2}+z^{\prime 2}+2\left(r^{2} \lambda^{\prime} \psi^{\prime}+z^{\prime} w^{\prime}\right) \xi+\left(r^{2} \lambda^{\prime 2}+w^{\prime 2}\right) \xi^{2} .
$$

The right side of (32) will be independent of $\xi$ only if $\lambda$ and $w$ are constants, and this also causes the last relation in (23) to be satisfied. With the change of variables $\theta=\lambda \xi+\psi(\eta), \phi$ $=\eta,(30)$ takes the form of (1), the canonical equation of a helicoid.

$\mathbf{w}=0$. In this case, the first two relations in (23) are satisfied identically. The next two relations imply that

$$
\mathbf{z}^{\prime}=\alpha(\eta) \mathbf{e}_{z}(\eta)
$$

where $\alpha$ is arbitrary and $\mathbf{e}_{\boldsymbol{z}}=\mathbf{e}_{\boldsymbol{x}} \times \mathbf{e}_{y}$. The last relation in (23) implies that $\lambda$ is a constant. As $\mathbf{e}_{x}$ and $\mathbf{e}_{y}$ are orthogonal unit vectors, we may set

$$
\begin{aligned}
\mathbf{e}_{x}^{\prime} & =\beta(\eta) \mathbf{e}_{y}(\eta)+\gamma(\eta) \mathbf{e}_{z}(\eta), \\
\mathbf{e}_{y}^{\prime} & =-\beta(\eta) \mathbf{e}_{x}(\eta)+\delta(\eta) \mathbf{e}_{z}(\eta),
\end{aligned}
$$

where $\beta, \gamma$, and $\delta$ at this point are arbitrary. This allows us to write (19) in the form

$$
\mathbf{e}_{r}^{\prime}=\beta \mathbf{e}_{\theta}+(\gamma \cos \lambda \xi+\delta \sin \lambda \xi) \mathbf{e}_{z} .
$$

Hence

$$
\begin{gathered}
\mathbf{r}(\xi, \eta)=r(\eta) \mathbf{e}_{r}(\xi, \eta)+\mathbf{z}(\eta), \\
\mathbf{r}^{\prime}=r^{\prime} \mathbf{e}_{r}+r \beta \mathbf{e}_{\theta}+[\alpha+r(\gamma \cos \lambda \xi+\delta \sin \lambda \xi)] \mathbf{e}_{z}
\end{gathered}
$$

Thus

$$
\begin{gathered}
a_{22}=\mathrm{r}^{\prime} \cdot \mathrm{r}^{\prime}=r^{2}+r^{2} \beta^{2}+\alpha^{2}+\frac{1}{2} r^{2}\left(\gamma^{2}+\delta^{2}\right) \\
+2 r \alpha \gamma \cos \lambda \xi+2 r \alpha \delta \sin \lambda \xi+\frac{1}{2} r^{2}\left(\gamma^{2}-\delta^{2}\right) \cos 2 \lambda \xi+r^{2} \gamma \delta \sin 2 \lambda \xi .
\end{gathered}
$$

The right side of (39) will be independent of $\xi$ only if $\gamma=\delta=0$. Thus (34) and (35) imply that $\mathbf{e}_{x}$ and $\mathbf{e}_{y}$ must, again, lie in a fixed plane and so may be represented by (26). We then have $\mathbf{e}_{z}=\mathbf{k}$ and (33) implies that $z$ is again of the form (29). Hence

$$
\mathbf{r}(\xi, \eta)=r(\eta) \mathbf{e}_{r}(\xi, \eta)+z(\eta) \mathbf{k}
$$

and with the change of variable $\theta=\lambda \xi+\psi(\eta), \phi=\eta,(40)$ again takes the form of (1), the canonical equation of a helicoid.

\section{REFERENCES}

[1] E. Reissner, On finite bending and twisting of circular ring sector plates and shallow helicoidal shells, Quart. Appl. Math. 11, 473-483 (1953)

[2] F. Y. M. Wan, personal communication, June 1978

[3] L. P. Eisenhart, A treatise on the differential geometry of curves and surfaces, Dover, New York, 1960

[4] D. J. Struik, Differential geometry, 2nd edition, Addison-Wesley, Reading, Mass., 1961 